Chinese Journal of Organic Chemistry

NOTE

\title{
石斛根中三个新的菲醌类化合物
}

\author{
刘英豪 $a, c$ 林芳霞 $a, c$ 谭银丰 $b$ 杨静雨 $a, c$ \\ 张斌 $a, c$ 周学明 $*, a, c$ 宋金金明 $*, a, c$ \\ ( ${ }^{a}$ 海南师范大学化学与化工学院 热带药用资源化学教育部重点实验室 海口 571158) \\ ( $b$ 海南医学院 海南省热带药用植物研究开发重点实验室 海口 570216) \\ ( $c$ 海南师范大学化学与化工学院 热带药用植物化学海南省重点实验室 海口 571158)
}

\begin{abstract}
摘要 综合运用多种现代色谱技术对兰科石斛属植物石斛 Dendrobium nobile Lindl.根中的化学成分进行了系统的分离 纯化, 从其根的乙醇提取物中分离得到了 phenanobiles $\mathrm{A} \sim \mathrm{C}(\mathbf{1} \sim \mathbf{3}), 6$,7-dihydroxy-2-methoxy-1,4-phenanthrenedione (4) 和 ephemeranthoquinone (5) 5 个菲醌类化合物. 采用多种波谱技术确定了这些化合物的结构, 其中化合物 $1 \sim 3$ 为新的 菲醌类化合物. 化合物 $\mathbf{1} \sim \mathbf{5}$ 的体外抗肿瘤活性评价结果表明它们对 3 种肿瘤细胞株(HL-60, A549 以及 MCF-7)显示出 体外生长抑制活性.
\end{abstract}

关键词 石斛属; 石斛; 菲醌; 抗肿瘤活性

\section{Three New Phenanthraquinones from the Root of Dendrobium nobile}

\author{
Liu, Yinghao ${ }^{a, c}$ \\ Lin, Fangxia ${ }^{a, c}$ \\ Tan, Yinfeng ${ }^{b}$ \\ Yang, Jingyu ${ }^{a, c}$ \\ Zhang, Bin ${ }^{a, c}$ \\ Zhou, Xueming ${ }^{*, a, c}$ \\ Song, Xinming ${ }^{*, a, c}$ \\ $\left({ }^{a}\right.$ Key Laboratory of Tropical Medicinal Resource Chemistry of Ministry of Education, College of Chemistry and \\ Chemical Engineering, Hainan Normal University, Haikou 571158) \\ ( ${ }^{b}$ Hainan Provincial Key Laboratory of Research and Development on Tropical Herbs, Hainan Medical College, \\ Haikou 570216) \\ ( ${ }^{c}$ Key Laboratory of Tropical Medicinal Plant Chemistry of Hainan Province, College of Chemistry and \\ Chemical Engineering, Hainan Normal University, Haikou 571158)
}

\begin{abstract}
Three new phenanthraquinones, phenanobiles $\mathrm{A} \sim \mathrm{C}(\mathbf{1} \sim \mathbf{3})$, together with two known phenanthraquinones 6,7-dihydroxy-2-methoxy-1,4-phenanthrenedione (4) and ephemeranthoquinone (5), were isolated from the root of Dendrobium nobile. Their structures were elucidated using comprehensive spectroscopic methods. The antitumor activities of compounds $\mathbf{1} \sim \mathbf{5}$ were evaluated against three cancer cell lines (HL-60, A549 and MCF-7). Compounds $\mathbf{1} \sim \mathbf{5}$ exhibited inhibitory effects against three cancer cell lines HeLa, MCF-7 and A549 in vitro.
\end{abstract}

Keywords Dendrobium; Dendrobium nobile; phenanthraquinone; antitumor activity

兰科(Orchidaceae)石斛属 (Dendrobium)植物全球 约有 1100 种, 主要分布于亚洲西南部、欧洲以及大洋洲, 我国有 74 种以及 2 个变种, 广泛分布于秦岭以南的各个 省 ${ }^{[1]}$. 石斛属植物由于其药用价值以及观赏价值而受 到国内外学者的广泛关注. 近年来, 许多植化研究学者
对该属植物进行了系统的活性成分研究，并分离鉴定了 许多具有显著生物活性的化合物，如菲类、二芳基乙烷 类、聚酮类、倍半萜类、生物碱类以及木脂素类等多种 类型的化合物 ${ }^{[2-9]}$. 石斛(Dendrobium nobile Lind1.)作为 一种观赏植物在海南被广泛地种植, 然而在其采收三到

\footnotetext{
* Corresponding authors. E-mail: xueming2009211@126.com; sxm8646@163.com

Received December 1, 2020; revised December 28, 2020; published online January 21, 2021.

Project supported by the Natural Science Foundation of Hainan Province (No. 218QN234), the Open Research Projects of Hainan Provincial Key Laboratory of Research and Development on Tropical Herbs (No. KF202002), the National Natural Science Foundation of China (Nos. 21662012, 41866005) and the Innovative Research Team Project of Ministry of Education (No. IRT-16R19).

海南省自然科学基金(No. 218QN234)、海南省热带药用植物研究开发重点实验室开放研究(No. KF202002)、国家自然科学基金(Nos. 21662012, 41866005)以及教育部创新研究团队(No. IRT-16R19)资助项目.
} 
四年后该植物的根茎就被废弃. 为了开发石斛的额外价 值, 本课题组前期对其茎的活性成分进行了系统的分离 并发现了一系列的菲类以及内脂类化合物, 其中的菲类 化合物表现出抗肿瘤以及抗植物病原菌活性 ${ }^{[5,10]}$. 为进 一步研究石斛的活性成分, 本研究对石斛根的 $95 \%$ 乙醇 提取物进行了抗肿瘤活性成分的追踪分离, 并分离得到 了 5 个菲醌类化合物, 分别鉴定为 phenanobiles $\mathrm{A} \sim \mathrm{C}$ $(\mathbf{1} \sim 3)$, 6,7-dihydroxy-2-methoxy-1,4-phenanthrenedione (4)和 ephemeranthoquinone (5), 其中 $1 \sim 3$ 为新的菲醌 类化合物(图 1). 此外, 我们对化合物 $\mathbf{1} \sim \mathbf{5}$ 的体外抗肿 瘤活性进行了评价, 结果表明化合物 1 5 对 HL-60, A549 以及 MCF-7 三种肿瘤细胞株均表现出生长抑制活 性.
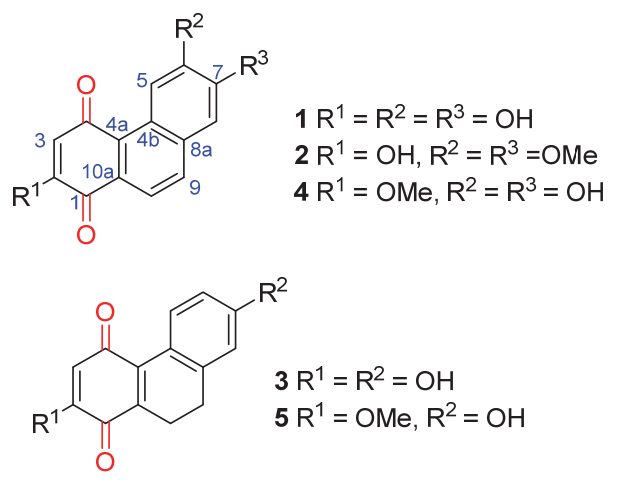

图 1 化合物 $1 \sim 5$ 的化学结构

Figure 1 Chemical structures of compounds $1 \sim 5$

\section{1 结果与讨论}

化合物 1 为深红色无定形粉末, HRESIMS 给出化合 物的 $[\mathrm{M}+\mathrm{H}]^{+}$峰为 $m / z: 257.0447$ (calcd for $\mathrm{C}_{14} \mathrm{H}_{9} \mathrm{O}_{5}$
$257.0444)$, 可以确定该化合物的分子式为 $\mathrm{C}_{14} \mathrm{H}_{8} \mathrm{O}_{5}$, 不 饱和度为 11. 在化合物 $\mathbf{1}$ 的氢谱中, 仅在低场区显示了 5 个芳香质子信号 $\left[\delta_{\mathrm{H}} 8.99(\mathrm{~s}, 1 \mathrm{H}), 8.02(\mathrm{~d}, J=8.8 \mathrm{~Hz}\right.$, $1 \mathrm{H}), 7.84(\mathrm{~d}, J=8.8 \mathrm{~Hz}, 1 \mathrm{H}), 7.26(\mathrm{~s}, 1 \mathrm{H})$ 和 $6.90(\mathrm{~s}, 1 \mathrm{H})]$ (表 1). 在化合物 $\mathbf{1}$ 的碳谱中, 所有信号都集中在低场区, 共 14 个信号分别为 2 个羰基碳信号 $\left(\delta_{\mathrm{C}} 188.8\right.$ 和 182.9) 以及 12 个芳香碳信号 $\left(\delta_{\mathrm{C}} 109.9 \sim 156.3\right)$. 这些数据与已 知化合物 4 的数据非常相似，对比化合物 $\mathbf{4}$ 的数据化合 物 1 只是缺少了一个甲氧基信号 $\left(\delta_{\mathrm{H}} 3.84\right.$ 和 $\left.\delta_{\mathrm{C}} 56.3\right)$, 这 说明在化合物 1 中 2 位连的为羟基而不是甲氧基. 同样 根据 HMBC 相关信号可以确定化合物 $\mathbf{1}$ 的结构. HMBC 谱中 $\mathrm{H}-3$ 与 $\mathrm{C}-1 / 2 / 4 / 4 \mathrm{a}$ 相关可以确定化合物 1 中具有一 个对苯醌结构(A 环)(图 2); H-10 与 C-10a/4a/9/8a 相关, H-9 与 C- $10 \mathrm{a} / 10 / 4 \mathrm{~b} / 8 \mathrm{a}$ 相关, H-5 与 C-6/7/4a/4b/8a 相关 以及 $H-8$ 与 C-6/7/9/4b/8a 相关可以确定化合物 1 中具有 一个菜环( $\mathrm{B}$ 和 $\mathrm{C}$ 环)结构并且 6 位和 7 位连有着基; H-10 与 $C-4 a / 10 a / 1$ 相关可以确定荎环通过 C-4a/10a 并在对苯 醌环上. 至此, 可以确定化合物 $\mathbf{1}$ 的结构如图 1 所示, 并 将化合物 1 命名为 phenanobile A.

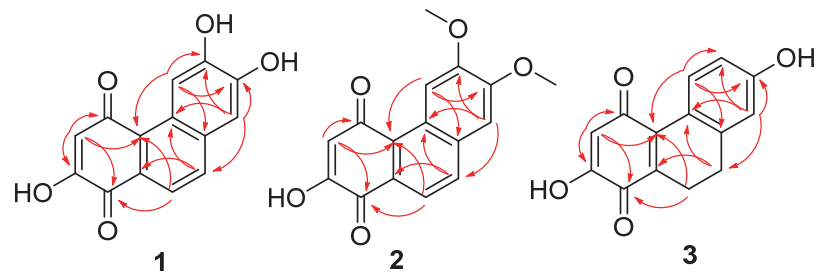

图 2 化合物 $\mathbf{1} \sim \mathbf{3}$ 的 $\mathrm{HMBC}$ 相关

Figure 2 HMBC correlations of compounds $1 \sim 3$

表 1 化合物 phenanobiles $\mathrm{A} \sim \mathrm{C}(\mathbf{1} \sim \mathbf{3})$ 的 ${ }^{1} \mathrm{H}$ NMR $(400 \mathrm{MHz})$ 和 ${ }^{13} \mathrm{C} \mathrm{NMR}(100 \mathrm{MHz})$ 数据

Table $1{ }^{1} \mathrm{H}$ NMR $(400 \mathrm{MHz})$ and ${ }^{13} \mathrm{C}$ NMR $(100 \mathrm{MHz})$ spectral data of phenanobiles $\mathrm{A} \sim \mathrm{C}(\mathbf{1} \sim \mathbf{3})$

\begin{tabular}{|c|c|c|c|c|c|c|}
\hline \multirow{2}{*}{ Position } & \multicolumn{2}{|l|}{1 (DMSO- $\left.d_{6}\right)$} & \multicolumn{2}{|l|}{2 (DMSO- $\left.d_{6}\right)$} & \multicolumn{2}{|l|}{$3\left(\mathrm{CD}_{3} \mathrm{OD}\right)$} \\
\hline & $\delta_{\mathrm{H}}(J$ in $\mathrm{Hz})$ & $\delta_{\mathrm{C}}$ & $\delta_{\mathrm{H}}(J$ in $\mathrm{Hz})$ & $\delta \mathrm{C}$ & $\delta_{\mathrm{H}}(J$ in $\mathrm{Hz})$ & $\delta_{\mathrm{C}}$ \\
\hline 1 & - & 182.9 & - & 180.2 & - & 183.3 \\
\hline 2 & - & 156.3 & - & 156.9 & - & 169.4 \\
\hline 3 & $6.90(\mathrm{~s}, 1 \mathrm{H})$ & 112.6 & $6.94(\mathrm{~s}, 1 \mathrm{H})$ & 111.3 & $5.93(\mathrm{~s}, 1 \mathrm{H})$ & 109.0 \\
\hline 4 & - & 182.9 & - & 188.2 & - & 189.6 \\
\hline $4 a$ & - & 124.7 & - & 124.1 & - & 137.7 \\
\hline $4 \mathrm{~b}$ & - & 125.5 & - & 124.9 & - & 122.6 \\
\hline 5 & $8.99(\mathrm{~s}, 1 \mathrm{H})$ & 109.9 & $8.87(\mathrm{~s}, 1 \mathrm{H})$ & 105.6 & $7.94(\mathrm{~d}, 9.2,1 \mathrm{H})$ & 133.7 \\
\hline 6 & - & 151.4 & - & 153.7 & $6.72(\mathrm{dd}, 9.2,2.4,1 \mathrm{H})$ & 115.2 \\
\hline 7 & - & 149.8 & - & 152.1 & - & 161.4 \\
\hline 8 & $7.26(\mathrm{~s}, 1 \mathrm{H})$ & 110.7 & $7.20(\mathrm{~s}, 1 \mathrm{H})$ & 107.2 & $6.71(\mathrm{~d}, 2.4,1 \mathrm{H})$ & 116.4 \\
\hline $8 \mathrm{a}$ & - & 128.9 & - & 128.3 & - & 138.8 \\
\hline 9 & $8.02(\mathrm{~d}, 8.8,1 \mathrm{H})$ & 132.0 & $8.11(\mathrm{~d}, 9.2,1 \mathrm{H})$ & 131.4 & $2.63 \sim 2.68(\mathrm{~m}, 2 \mathrm{H})$ & 21.7 \\
\hline 10 & $7.84(\mathrm{~d}, 8.8,1 \mathrm{H})$ & 119.4 & $7.61(\mathrm{~d}, 9.2,1 \mathrm{H})$ & 118.7 & $2.73 \sim 2.77(\mathrm{~m}, 2 \mathrm{H})$ & 29.1 \\
\hline $10 \mathrm{a}$ & - & 134.3 & - & 133.7 & - & 146.6 \\
\hline 6-OMe & - & - & $3.85(\mathrm{~s}, 3 \mathrm{H})$ & 56.0 & - & - \\
\hline 7-OMe & - & - & $3.82(\mathrm{~s}, 3 \mathrm{H})$ & 56.1 & - & - \\
\hline
\end{tabular}


化合物 2 同为深红色无定形粉末, 根据 HRESIMS 显示 的 $[\mathrm{M}+\mathrm{H}]^{+}$峰为 $m / z: 285.0751$ (calcd for $\mathrm{C}_{16} \mathrm{H}_{13} \mathrm{O}_{5}$ 285.0757 ), 可以确定该化合物的分子式为 $\mathrm{C}_{16} \mathrm{H}_{12} \mathrm{O}_{5}$, 不 饱和度也为 11. 在化合物 2 的氢谱中, 在低场区同样显 示有 5 个芳香质子信号 $\left[\delta_{\mathrm{H}} 8.87(\mathrm{~s}, 1 \mathrm{H}), 8.11(\mathrm{~d}, J=9.2\right.$ $\mathrm{Hz}, 1 \mathrm{H}), 7.61(\mathrm{~d}, J=9.2 \mathrm{~Hz}, 1 \mathrm{H}), 7.20(\mathrm{~s}, 1 \mathrm{H})$ 和 6.94 (s, $1 \mathrm{H})$ ], 在在高场区显示有 2 个甲氧基信号 $[3.85(\mathrm{~s}, 3 \mathrm{H})$ 和 $3.82(\mathrm{~s}, 3 \mathrm{H})]$. 在化合物 2 的碳谱中, 共 16 个信号分别为 2 个羰基碳信号 $\left(\delta_{\mathrm{C}} 188.2\right.$ 和 180.2)、12 个芳香碳信号 $\left(\delta_{\mathrm{C}}\right.$ 105.6 156.9) 以及 2 个甲氧基碳信号 $\left(\delta_{\mathrm{C}} 56.1\right.$ 和 56.0). 这些数据表明化合物 2 也是菲醌类化合物, 详细对比化 合物 2 与 1 的数据发现化合物 2 对比化合物 1 只是多出 了两个甲氧基信号 $\left(\delta_{\mathrm{H}} 3.85\right.$ 和 3.82; $\delta_{\mathrm{C}} 56.1$ 和 56.0). 在 $\mathrm{HMBC}$ 谱中, $\mathrm{H}-5$ 与 $\mathrm{C}-7, \mathrm{H}-8$ 与 C-6, 6-OMe 与 C-6 以及 7-OMe 与 C-7 分别相关表明两个甲氧基分别连接在 C-6 和 C-7 位. 至此, 可以确定化合物 2 的结构如图 1 所示, 并将化合物 2 命名为 phenanobile B.

化合物 3 为红色无定形粉末, 根据 HRESIMS 显示 的 $[\mathrm{M}+\mathrm{H}]^{+}$峰为 $m / z: 243.0657$ (calcd for $\mathrm{C}_{14} \mathrm{H}_{11} \mathrm{O}_{4}$ 243.0652 ), 可以确定该化合物的分子式为 $\mathrm{C}_{14} \mathrm{H}_{10} \mathrm{O}_{4}$, 不 饱和度为 10. 在化合物 3 的氢谱中, 在低场区显示有 4 个芳香质子信号 $\left[\delta_{\mathrm{H}} 7.94(\mathrm{~d}, J=9.2 \mathrm{~Hz}, 1 \mathrm{H}), 6.72(\mathrm{dd}, J=\right.$ 9.2, $2.4 \mathrm{~Hz}, 1 \mathrm{H}), 6.71(\mathrm{~d}, J=2.4 \mathrm{~Hz}, 1 \mathrm{H})$ 和 $5.93(\mathrm{~s}, 1 \mathrm{H})]$, 在高场区显示有 2 个亚甲基信号 $[2.73 \sim 2.77(\mathrm{~m}, 2 \mathrm{H})$ 和 $2.63 \sim 2.68(\mathrm{~m}, 2 \mathrm{H})]$. 在化合物 3 的碳谱中, 共 14 个信号 分别为 2 个羰基碳信号 $\left(\delta_{\mathrm{C}} 189.6\right.$ 和 183.3)、10 个芳香碳 信号 $\left(\delta_{\mathrm{C}} 109.0 \sim 169.4\right)$ 以及 2 个亚甲基碳信号 $\left(\delta_{\mathrm{C}} 29.1\right.$ 和 21.7). 这些数据表明化合物 3 同为菲醌类化合物, 只是 9 位双键被还原了变成 9,10-二氢菲醌. 上述化合物 3 的 数据与已知化合物 $\mathbf{5}$ 的数据非常相似, 详细对比化合物 $\mathbf{3}$ 与 $\mathbf{5}$ 的数据发现化合物 $\mathbf{3}$ 对比化合物 $\mathbf{5}$ 只是少了一个 甲氧基信号 $\left(\delta_{\mathrm{H}} 3.85\right)$, 这说明在化合物 3 中 2 位连的为 羟基而不是甲氧基. 详细分析 $\mathrm{HMBC}$ 同样确定该化合 物结构如图 1 所示, 我们将化合物 $\mathbf{3}$ 命名为 phenanobile C.

经 NMR 以及 MS 分析并与文献中相应化合物的数 据进行对照, 将化合物 4 和 5 分别鉴定为 6,7-dihydroxy2-methoxy-1,4-phenanthrenedione $(4)^{[11]}$ 和 ephemeranthoquinone (5) ${ }^{[12]}$.

采用与之前报道所用一致的噻唑兰(MTT)法 ${ }^{[5]}$, 对 5 个化合物进行了体外抗肿瘤活性测试, 肿瘤细胞株分 别为 HL-60, A549 以及 MCF-7, 以阿霉素为阳性对照药 物. 测试结果表明它们对 3 中肿瘤细胞株均显示出体外 生长抑制活性(表 2), 其中化合物 1 对 A549 细胞株表现 出显著的生长抑制活性, 其 $\mathrm{IC}_{50}$ 值与阳性对照相当.
表 2 化合物 1 5 对肿瘤细胞的生长抑制活性

Table 2 Inhibitory activity of compounds $\mathbf{1} \sim \mathbf{5}$ on tumor cells

\begin{tabular}{cccc}
\hline \multirow{2}{*}{ Compd. } & \multicolumn{3}{c}{$\mathrm{IC}_{50} /\left(\mu \mathrm{mol} \cdot \mathrm{L}^{-1}\right)$} \\
\cline { 2 - 4 } & HL-60 & A549 & MCF-7 \\
\hline $\mathbf{1}$ & $5.21 \pm 0.17$ & $3.21 \pm 0.12$ & $9.52 \pm 0.11$ \\
$\mathbf{2}$ & $15.18 \pm 0.14$ & $21.56 \pm 0.08$ & $31.51 \pm 0.13$ \\
$\mathbf{3}$ & $11.28 \pm 0.09$ & $30.12 \pm 0.06$ & $29.12 \pm 0.10$ \\
$\mathbf{4}$ & $8.99 \pm 0.11$ & $21.03 \pm 0.12$ & $30.88 \pm 0.12$ \\
$\mathbf{5}$ & $86 \pm 0.14$ & $31.11 \pm 0.11$ & $28.89 \pm 0.09$ \\
阿霉素 & $0.36 \pm 0.11$ & $2.18 \pm 0.08$ & $5.66 \pm 0.12$ \\
\hline
\end{tabular}

\section{2 实验部分}

\section{1 仪器与试剂}

Bruker AV-400 型超导核磁共振仪(德国布鲁克公 司); Finnigan LCQ Advantage MAX 型质谱仪(美国热电 公司); Agilent 1200 型半制备高效液相色谱仪(美国安捷 伦科技有限公司); SANYO MCO-18AIC 二氧化碳培养 箱(日本 Sanyo 公司); 自动旋浴振荡器(上海博迅实业有 限公司); PHS-25 型酸度计(上海精密科学仪器有限公 司); 倒置显微镜(日本 OLYMPUS 公司); 64R 型低温高 速离心机(美国 BECKMAN 公司); infinite 200Pro 多功能 酶标仪(TECAN); Cosmosil C18 半制备型色谱柱(250 $\mathrm{mm} \times 10.0 \mathrm{~mm}, 5 \mu \mathrm{m})$. 薄层硅胶 GF254 以及柱色谱硅胶 (100～200、200～300 以及 300～400 目)购买于青岛海 洋化工厂; Sephadex LH-20 凝胶购买于 Amersham Blosclences 公司; ODS 柱色谱材料(C18，10４0 $\mu \mathrm{m})$ 购 买于 Merck 公司. 用于高效液相的甲醇与乙腈均为色 谱纯试剂, 其他有机试剂均为分析纯试剂. 96 孔板购买 于 Corning Costar (Cambridge, MA, USA)公司.

\section{2 材料}

石斛根于 2018 年 7 月采集的海南省海口市云龙镇 的农户种植的石斛, 由海南师范大学生命科学院钟琼芯 教授鉴定为石斛根, 其凭证标本(No. SHG20180712)保 存于海南师范大学热带药用资源化学教育部重点实验 室标本室.

\section{3 提取与分离}

将采集的石斛根(湿样 $2.5 \mathrm{~kg}$ )剪碎，用 $95 \%$ 的乙醇 室温浸泡提取(7 d X 3$)$, 按照常规浓缩萃取得到石油醚 部位(24.5 g)以及乙酸乙酯部位(32.1 g). 经活性测试表 明石油醚部位具有一定的抗肿瘤活性，选石油醚部位经 用 $100 \sim 200$ 目硅胶拌样, 采用硅胶常压柱(柱填料为 $200 \sim 300$ 目硅胶) 依次用石油醚/乙酸乙酯 $(V: V=100$ : $0 \sim 0 ： 100$ )进行梯度洗脱. 经薄层色谱(TLC)检测后合 并相似流分后得 5 个组分 $(F r .1 \sim$ Fr.5). Fr.2 (约 $2.8 \mathrm{~g}$ ) 组分 经凝胶 Sephadex LH-20 柱层析(洗脱剂: 氯仿甲醇等体 积混合液)得到各流分, 各流分再经 TLC 检测后合并相 
似流分后得 3 个小组分(Fr.2a Fr.2c). Fr.2b 由半制备高 效液相色谱(采用洗脱条件为: 乙腈/水, $V: V=55: 45$, 流速 $3 \mathrm{~mL} / \mathrm{min}$ )进行纯化得到化合物 1 (3 mg)(保留时间 $11.3 \mathrm{~min}) 、 2(9 \mathrm{mg}$ )(保留时间 $17.2 \mathrm{~min}$ )和 $4(4 \mathrm{mg})$ (保留 时间 $15.9 \mathrm{~min}$ ). Fr.2c 由 ODS 色谱柱(采用洗脱条件为: 甲醇/水, $V: V=40: 60$, 流速 $3 \mathrm{~mL} / \mathrm{min}$ )进行纯化得到 化合物 $3(8 \mathrm{mg})$ (保留时间 $13.8 \mathrm{~min}$ )和 $\mathbf{5}(12 \mathrm{mg})$ (保留时 间 $16.2 \mathrm{~min})$.

Phenanobile A (1): 深红色无定形粉末. m.p. 269

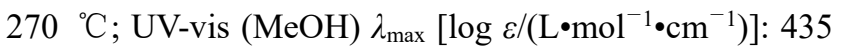
(2.62), 286 (3.10), 247 (3.32) nm; ${ }^{1} \mathrm{H}$ NMR (400 MHz, DMSO- $\left.d_{6}\right)$ 和 ${ }^{13} \mathrm{C}$ NMR $\left(100 \mathrm{MHz}\right.$, DMSO- $\left.d_{6}\right)$ 数据见表 1; IR (KBr) $v_{\max } 3356,2932,1678,1609 \mathrm{~cm}^{-1}$; HRESIMS calcd for $\mathrm{C}_{14} \mathrm{H}_{9} \mathrm{O}_{5} 257.0444$, found 257.0447.

Phenanobile B (2): 深红色无定形粉末. m.p. 266

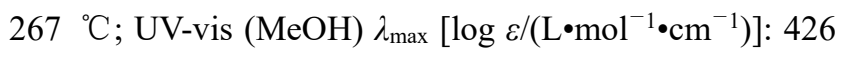
(2.33), 276 (3.08), 236 (3.27) nm; ${ }^{1} \mathrm{H}$ NMR (400 MHz, DMSO- $\left.d_{6}\right)$ 和 ${ }^{13} \mathrm{C}$ NMR (100 MHz, DMSO- $\left.d_{6}\right)$ 数据见表 1; IR (KBr) $v_{\max } 3341,2927,1675,1611 \mathrm{~cm}^{-1}$; HRMS calcd for $\mathrm{C}_{16} \mathrm{H}_{13} \mathrm{O}_{5}$ 285.0757, found 285.0751.

Phenanobile C (3): 深红色无定形粉末. m.p. 255

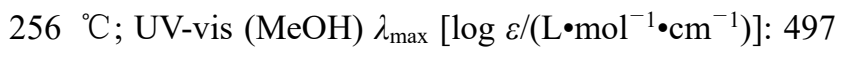
(2.87), 336 (3.61), 268 (3.89) nm; ${ }^{1} \mathrm{H}$ NMR (400 MHz, $\mathrm{CD}_{3} \mathrm{OD}$ ) 和 ${ }^{13} \mathrm{C} \mathrm{NMR}\left(100 \mathrm{MHz}, \mathrm{CD}_{3} \mathrm{OD}\right.$ )数据见表 1; IR (KBr) $v_{\max } 3346,2925,1617 \mathrm{~cm}^{-1}$; HRMS (ESI) calcd for $\mathrm{C}_{14} \mathrm{H}_{11} \mathrm{O}_{4} 243.0652$, found 243.0657 .

\section{4 抗肿瘤活性评价}

采用 MTT 法评价了化合物 1 5 对 HL-60, A549 以 及 MCF-7 三种肿瘤细胞株生长抑制活性. 化合物以固 定浓度 $40 \mu \mathrm{mol} \cdot \mathrm{L}^{-1}$ 进行初篎, 在该浓度对肿瘤细胞生
长抑制率在 $50 \%$ 以上的化合物设 5 个浓度进入梯度复 筛, 以阿霉素为阳性对照药. 每组实验平行三次, 所测 数据以浓度为横坐标, 细胞存活率为纵坐标绘制细胞生 长曲线, 应用两点法计算化合物的 $\mathrm{IC}_{50}$ 值.

辅助材料(Supporting Information) 化合物 phenanobiles $\mathrm{A} \sim \mathrm{C}(\mathbf{1} \sim \mathbf{3})$ 的 ${ }^{1} \mathrm{H}$ NMR、 ${ }^{13} \mathrm{C}$ NMR、HSQC, HMBC 和 HRESIMS 谱. 这些材料可以免费从本刊网站(http:// sioc-journal.cn/)上下载.

\section{References}

[1] Jiangsu New Medical College Dictionary of Chinese Medicines, Shanghai Scientific and Technologic Press, Shanghai, 1986, p. 586 (in Chinese).

(江苏新医学院, 中药大辞典, 上海科学技术出版社, 上海, 1986, p. 586.)

[2] Zhang, M. S.; Lang, H. L.; Zhang, J. Y.; Nie, X. Q.; Li, X. F.; Guo, D. L.; Xiao, S. J. Chin. J. Org. Chem. 2019, 39, 3289 (in Chinese). (张茂生, 令狐浪, 张建永, 聂绪强, 李晓飞, 郭大乐, 肖世基, 有机化学, 2019, 39, 3289.)

[3] Ma, C.; Meng, C. W.; Zhou, Q. M.; Peng, C.; Liu, F.; Zhang, J. W.; Zhou, F.; Xiong, L. Fitoterapia 2019, 138, 104351.

[4] Hu, M.; Yang, X. Q.; Wan, C. P.; Wang, B. Y.; Yin, H. Y.; Shi, L. J.; Wu, Y. M.; Yang, Y. B.; Zhou, H.; Ding, Z. T. RSC Adv. 2018, 8, 41810.

[5] Zhou, X. M.; Zheng, C. J.; Gan, L. S.; Chen, G. Y.; Zhang, X. P.; Song, X. P.; Li, G. N.; Sun, C. G. J. Nat. Prod. 2016, 79, 1791.

[6] Zhang, X.; Tu, F. J.; Yu, H. Y.; Wang, N. L.; Wang, Z.; Yao, X. S. Chem. Pharm. Bull. 2008, 56, 854.

[7] Yang, H.; Sung, S. H.; Kim, Y. C. J. Nat. Prod. 2007, 70, 1925.

[8] Xu, X. L.; Chen, X. Y.; Yang, R. M.; Li, Z. S.; Zhou, H. G.; Bai, Y. B.; Yu, M.; Li, B.; Ding, G. Sci. Rep. 2020, 10, 9564.

[9] Yang, D.; Cheng, Z. Q.; Huo, B.; Yang, L.; Zi, C. T.; Dong, F. W.; Hu, J. M.; Zhou, J. Fitoterapia 2020, 144, 104607.

[10] Zhou, X. M.; Zheng, C. J.; Wu, J. T.; Chen, G. Y.; Chen, J.; Sun, C. G. Fitoterapia 2016, 115, 96.

[11] Ma, C.; Wang, W.; Chen, Y. Y.; Liu, R. N.; Wang, R. F.; Du, L. J. J. Nat. Prod. 2005, 68, 1259.

[12] Tezuka, Y.; Hirano, H.; Kikuchi, T.; Xu, G. J. Chem. Pharm. Bull. 1991, 39, 593. 